\title{
Genomic characterization of DEP1 gene in the Triticinae species with compact, compactoid and normal spike shape
}

\author{
V. Vavilova*, I. Konopatskaia, A. Blinov \\ Institute of Cytology and Genetics SB RAS, Novosibirsk, Russia \\ *e-mail:valeriya-vavilova@bionet.nsc.ru
}

Key words: $D E P 1$ gene, Triticinae, Triticum, Aegilops, spike shape, plant architecture

Motivation and Aim: Spike shape of Triticinae species is one of the most important taxonomically characteristic of this tribe. There are four main variants of spike shape (spelt, compact, compactoid and normal), that widely distributed among wheat and Aegilops species. Several genes and loci associated with spike shape trait have been identified previously [1]. Nevertheless, there are practically no data about nucleotide sequences of this genes and loci. DENSE AND ERECT PANICLE 1 (DEP1) gene is related to several traits in rice (erect panicle, number of grains per panicle and panicle dense) [2]. In case of $T$. aestivum the experiments with the transgenic line showed that downregulation of DEP1 homologue affects the length of the ear, ear density and number of spikelets [3].The aim of this study was investigation and genomic characterization of DEP1 gene in wheat and Aegilops species with compact, compactoid and normal spike shape.

Methods and Algorithms: A combination of bioinformatical tools and standard molecular biology methods was used.

Results: We determined the spike shape of several accessions of Triticum and Aegilops species (T. antiquorum, T. macha, T. sphaerococcum and Ae. tauschii) by calculation of Flaksberger's formula. The full-length sequences of DEP1 gene were obtained for all accessions studied. Nucleotide sequences comparing revealed DEP1 gene regions which distinguish analyzed wheat and Aegilops species with compact, compactoid and normal spike phenotypes. Phylogenetic analysis allowed to determined the origin of DEP1 alleles and different Triticinae species.

Conclusion: DEP1 gene variability could contribute to the spike shape formation in wheat and Aegilops species.

Acknowledgements: The study was supported by the Russian Science Foundation (grant number: 16-16-10021).

References

1. Konopatskaia I. et al. (2016) Spike morphology genes in wheat species (Triticum L.). Proc. Latv. Acad. Sci. Sect. B. 70:345-355.

2. Huang X. et al. (2009) Natural variation at the DEP1 locus enhances grain yield in rice. Nat. Genet. 41:494-497.

3. Xu H. et al. (2016) The DENSE AND ERECT PANICLE 1 (DEP1) gene offering the potential in the breeding of high-yielding rice. Breed Sci. 66:659-667. 